\title{
Increased levels of circulating 8-oxodeoxyguanosine as a biomarker of sepsis severity in mice
}

\author{
Jun-ichi Hirata ${ }^{1 *}$, Munehiko Ohya ${ }^{1}$, Toshiomi Okuno ${ }^{2}$ and Keiji Kumon ${ }^{1}$
}

\begin{abstract}
Background: In intensive care for sepsis, damage of intracellular environmental states is responsible for not only infectious disease severity but also oxidative DNA damage following hypometabolism such as hypoxia, hypotension, hypothermia, and hypoglycemia. Therefore, identification of a biomarker to monitor these intracellular equilibrium conditions is crucial for the prognosis of disease severity. Under an environmental condition, the 8-oxodeoxyguanosine $(8-\mathrm{OH}-\mathrm{dG})$ levels in tissues were previously shown to be increased as a result of oxidative DNA damage. The purpose of this study was to elucidate whether the circulating $8-\mathrm{OH}-\mathrm{dG}$ levels may serve as a marker for severity in a mouse septic model.

Methods: Thirty young male C57BL/6 mice were subjected to sepsis induction by cecal ligation and puncture (CLP), whereas 10 control mice without sepsis were used as the sham group. Aspirated blood was obtained from either surviving mice at $72 \mathrm{~h}$ after sepsis induction or non-surviving mice at the time of death. The levels of circulating 8-OH-dG, serum lactate, and C-reactive protein (CRP) were determined by high-performance liquid chromatography. These markers were analyzed for their ability to indicate severity by calculating the area under the receiver operating characteristic (ROC) curve.

Results: The circulating 8-OH-dG levels, similar to the lactate levels, were significantly increased in the non-surviving group. The area under the ROC curve was $0.97 \pm 0.032$ and $0.92 \pm 0.061$ for $8-\mathrm{OH}-\mathrm{dG}$ and lactate, respectively, with the optimal threshold value to discriminate between the survivors and non-survivors being 1.3 (sensitivity, 80\%; specificity, 100\%) for 8-OH-dG and 14.1 (sensitivity, 70\%; specificity, 80\%) for lactate. No significant change in CRP was observed between the two groups. Circulating 8-OH-dG levels better indicated severity than lactate and CRP concentrations, strongly implicating circulating $8-\mathrm{OH}-\mathrm{dG}$ in intracellular environmental conditions in a mouse septic model.
\end{abstract}

Conclusion: The circulating 8-OH-dG levels may be a novel marker for evaluating disease severity during intensive care for sepsis.

Keywords: Circulating 8-oxodeoxyguanosine, Lactate, C-reactive protein, Oxidative DNA damage, Sepsis

\section{Background}

Intensive care following whole therapy for circulating, respiratory, and metabolic failure for septic patients in intensive care units comprises several approaches for maintaining intracellular steady-state conditions. In particular, intracellular ischemic conditions are caused easily by inadequate fluid, oxygen, antibiotic, or metabolic therapies, according to the clinical consequence of severe

\footnotetext{
* Correspondence: qq-hira@hyo-med.ac.jp

'Department of Emergency and Critical Care Medicine, Kinki University

Faculty of Medicine, Nara Hospital, Nara 630-0293, Japan

Full list of author information is available at the end of the article
}

sepsis and septic shock. Therefore, a biomarker for monitoring intracellular equilibrium conditions is crucial for evaluating not only disease severity but also therapeutic effects and self-recovery.

C-reactive protein (CRP) is a conventional marker that has a characteristic of reflecting the severity of infectious diseases such as pneumonia, pancreatitis, and sepsis [1]. This type of marker, though also used for the evaluation of antibiotic therapeutic effects, is limited for intensive care of hypometabolism such as hypoxia, hypotension, hypothermia, and hypoglycemia. 
We hypothesized that intracellular equilibrium conditions are subject to ischemic changes following oxidative DNA damage due to circulatory, respiratory, and metabolic failure. Therefore, the severity of oxidative DNA damage may serve as a marker of intensive care therapeutic effects on sepsis, severe sepsis, and septic shock [2].

We hypothesized that circulating 8-oxodeoxyguanosine (8-OH-dG) might be a crucial prognostic biomarker of mortality in sepsis. The purpose of this study was to elucidate whether the circulating $8-\mathrm{OH}-\mathrm{dG}$ level is a better severity marker than CRP. We also compared the ability of circulating 8-OH-dG and lactate levels to reflect intracellular ischemic conditions in a septic rat model.

\section{Methods}

\section{Animals}

All animal studies were approved by the Animal Studies Committee of the Hyogo College of Medicine. Animals were handled in accordance with the National Institutes of Health Guide for the Care and Use of Laboratory Animals and the Guidelines for Animal Experiments of Hyogo College of Medicine. Animals were anesthetized with pentobarbital $(50 \mathrm{mg} / \mathrm{kg}$, intraperitoneal administration (i.p.)) purchased from Wako Pure Chemical Industries, Ltd. (Osaka, Japan). A laparotomy was performed through a middle incision, and the abdomen was closed in two layers. All procedures were performed using aseptic techniques.

\section{Experimental groups}

Forty young male C57BL/6 mice weighing 25-30 g were divided into two groups as follows: mice undergoing a laparotomy either with (sepsis group, $n=30$ ) or without (sham group, $n=10$ ) sepsis induction.

\section{Sepsis induction}

Polymicrobial sepsis was induced by cecal ligation and puncture (CLP) as described by Rittirsch et al. [3]. In brief, the cecum was ligated below the ileocecal valve after midline laparotomy, followed by a through-andthrough needle puncture of the cecum with a 21-gauge needle. The cecum was gently squeezed to extrude adequate amounts of stool and then replaced into the peritoneal cavity. Ligation was performed halfway between the distal pole and the base of the cecum. The survival rate of such a procedure was reported to be $40 \%$. At $72 \mathrm{~h}$ after sepsis induction, mice were anesthetized by ether inhalation and sacrificed by exsanguination via cardiac puncture. Aspirated blood samples from the heart were immediately collected and assessed for the levels of circulating 8-OH-dG by high-performance liquid chromatography (HPLC), and lactate and CRP levels were measured at each hospital using commercially available kits and instruments.

\section{8-OH-dG assay}

Blood samples were obtained from the heart at $72 \mathrm{~h}$ after sepsis induction (mice were anesthetized by ether inhalation and sacrificed by exsanguination via cardiac puncture). Blood samples were centrifuged at 3,000 rpm for $3 \mathrm{~min}$, and the plasma was stored at $-80^{\circ} \mathrm{C}$ until the $8-\mathrm{OH}-\mathrm{dG}$ assay. For the 8-OH-dG assay, $200 \mu \mathrm{l}$ of pure water and $50 \mu \mathrm{l}$ of $20 \%$ sulfosalicylic acid were added to $300 \mu \mathrm{l}$ of plasma. Then, the mixture was filtered through a $0.22-\mu \mathrm{m}$ filter, and $50 \mu \mathrm{l}$ of the filtrate was applied to a Hitachi 853 amino acid analyzer (Hitachi, Tokyo, Japan) for measurement of $8-\mathrm{OH}-\mathrm{dG}$. The sensitivity for the 8-OH-dG measurement was $0.1 \mathrm{nmol} / \mathrm{ml}$.

\section{Statistical analysis and data presentation}

The cumulative probability of survival was assessed by the Kaplan-Meier method, and differences in survival were compared using the log-rank test. The area under the receiver operating characteristic (ROC) curve (AUC), sensitivity, and specificity were also evaluated by constructing ROC curves. ANOVA and Newman-Keuls tests were used for multiple comparisons. The correlations were examined using the Pearson correlation coefficient. $p$ values of $<0.05$ were considered significant. All values are expressed as means \pm standard error of the mean.

\section{Results}

The survival analysis was performed for the two groups of mice, sham $(n=10)$ and sepsis $(n=30)$ groups, during the 72 -h study period. The survival rate of the sepsis group was $43.1 \%$, similar to that reported by Rittirsch et al. [3].

We first investigated the difference in the circulating 8-OH-dG levels between the survivors and nonsurvivors ( $n=15$ each). Figure 1a shows the circulating 8-OH-dG levels in surviving mice and non-surviving mice at $72 \mathrm{~h}$ after sepsis induction. While $8-\mathrm{OH}-\mathrm{dG}$ was not found in any blood sample of 10 sham mice $(<0.1 \mathrm{~g} / \mathrm{ml}$; data not shown), it was detected in all the blood samples from mice with sepsis induction. The circulating $8-\mathrm{OH}-$ $\mathrm{dG}$ concentrations of the non-survivors were significantly higher than those of the survivors $(1.64 \pm 0.37 \mathrm{~g} / \mathrm{ml}$ vs. $0.92 \pm 0.21 \mathrm{~g} / \mathrm{ml}$; Table 1 ).

Next, to compare the changes of circulating 8-OH-dG levels with those of traditional biomarkers for the survivors and/or non-survivors, the levels of circulating lactate and CRP in the same samples were measured at each hospital using commercially available kits and instruments. Although no significant change was observed between the CRP levels in the survivors and nonsurvivors $(9.48 \pm 3.63 \mathrm{mg} / \mathrm{dl}$ vs. $12.06 \pm 3.04 \mathrm{mg} / \mathrm{dl}$; $p=0.054$ ), the latter was slightly higher (Figure $1 \mathrm{~b}$ and Table 1). Moreover, the lactate levels in the nonsurvivors were significantly higher than those in the 

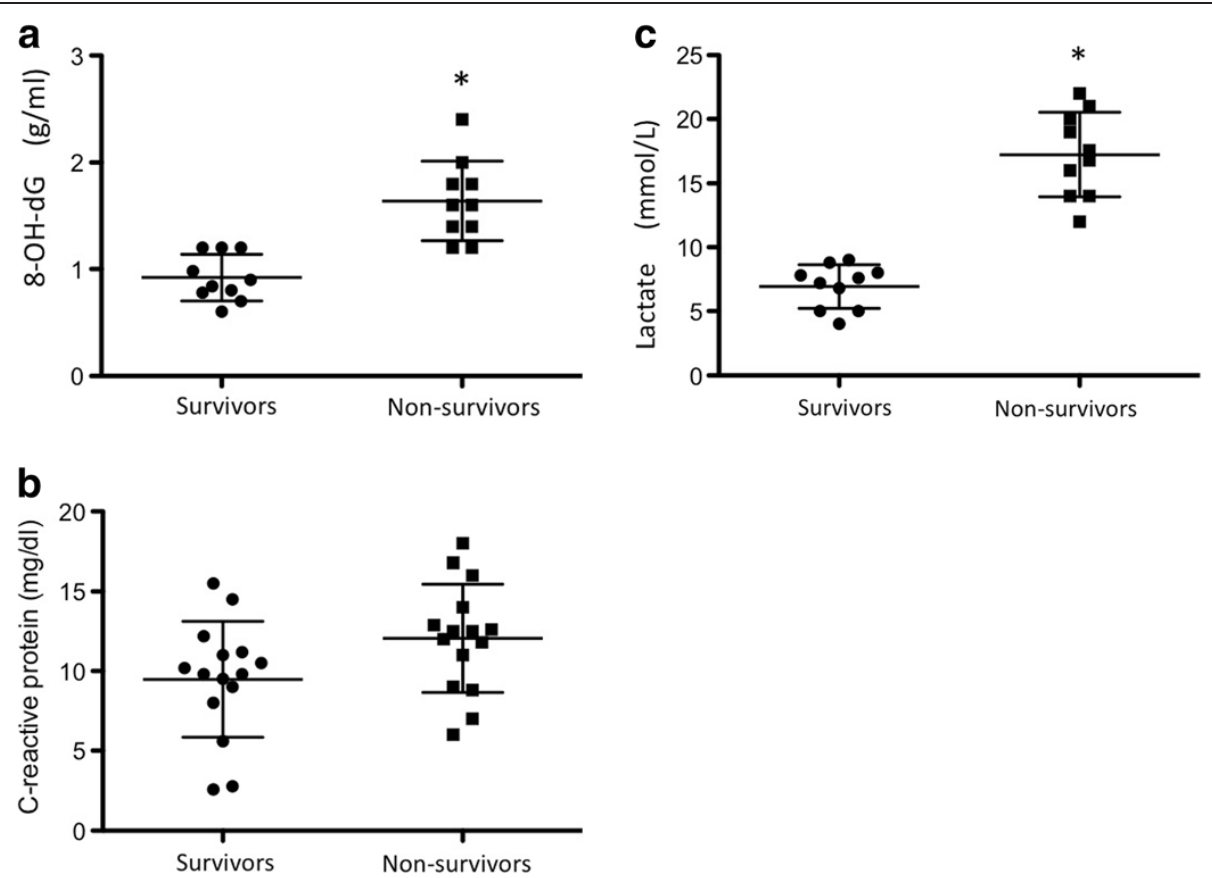

Figure 1 Comparison of circulating 8-OH-dG, CRP, and lactate levels between surviving and non-surviving septic mice. (a) Circulating 8-OH-dG levels between surviving and non-surviving septic mice. The circulating 8-OH-dG levels in the blood samples collected from surviving mice $72 \mathrm{~h}$ after CLP $(n=9)$ and non-surviving mice at the time of death (CLP $=21)$ were determined by high-performance liquid chromatography. Data represent means \pm standard error of the mean (SEM) of at least six independent experiments. ${ }^{*} p<0.05$ vs. the surviving group. (b) Serum C-reactive protein levels between surviving and non-surviving septic mice. Serum C-reactive protein concentrations in the blood samples from surviving mice $72 \mathrm{~h}$ after CLP $(n=9)$ and non-surviving mice at the time of death (CLP $=21)$ were evaluated. Data represent means \pm SEM of at least six independent experiments. (c) Serum lactate levels between surviving and non-surviving septic mice. Serum lactate concentrations in the blood samples prepared from surviving mice $72 \mathrm{~h}$ after CLP $(n=9)$ and non-surviving mice at the time of death $(C L P=21)$. Data represent means \pm SEM of at least six independent experiments. ${ }^{*} p<0.05$ vs. the surviving group.

survivors (17.24 \pm 3.30 vs. $10.00 \pm 3.63 ; p<0.05$; Figure $1 \mathrm{c}$ and Table 1). The AUC, sensitivity, and specificity were then evaluated based on the constructed ROC curves.

Table 2 summarizes the cutoff values, AUC, and the predictive accuracy of all three markers for the diagnosis of survivors and non-survivors in sepsis. With a cutoff point of 1.3 , the circulating $8-\mathrm{OH}-\mathrm{dG}$ level was found to be the most discriminatory laboratory variable with the highest sensitivity and specificity among all variables. When 1.09 was used as the cutoff value for $8-\mathrm{OH}$-dG, the sensitivity and specificity were $100.0 \%$ and $70 \%$, respectively; when the cutoff was 1.70 , these values were $40 \%$ and $100 \%$, respectively (data not shown). By the AUC analysis, 8-OH-dG was also the highest among all

Table 1 Comparison between circulating markers of survivors and non-survivors

\begin{tabular}{lccc}
\hline Variable & Survivors $(\boldsymbol{n}=\mathbf{1 5})$ & Non-survivors $(\boldsymbol{n}=\mathbf{1 5})$ & $\boldsymbol{p}$ value \\
\hline 8-OH-dG $(\mathrm{g} / \mathrm{ml})$ & $0.92 \pm 0.21$ & $1.64 \pm 0.37$ & $<0.05$ \\
Lactate $(\mathrm{mmol} / \mathrm{l})$ & $10.00 \pm 3.63$ & $17.24 \pm 3.30$ & $<0.05$ \\
CRP $(\mathrm{mg} / \mathrm{dl})$ & $9.48 \pm 3.63$ & $12.06 \pm 3.04$ & 0.054 \\
\hline
\end{tabular}

variables $(0.97 \pm 0.032$; $95 \%$ confidence interval, 0.907 1.033; Figure 2a,b,c). Of particular interest were the low AUC, sensitivity, and specificity in CRP (AUC, 0.71; $p=0.05$; sensitivity, 66.7 ; specificity, 80.0 ), compared to the other variables. Importantly, the parameters of both 8-OH-dG and lactate were significantly discriminatory between the two groups (survivors vs. non-survivors).

\section{Discussion}

To the best of our knowledge, this report is the first to demonstrate increased levels of circulating $8-\mathrm{OH}-\mathrm{dG}$ in

Table 2 Circulating parameters of the sepsis group $72 \mathbf{h}$ after cecal ligation and puncture

\begin{tabular}{lccccc}
\hline & $\begin{array}{c}\text { Best } \\
\text { cutoff }\end{array}$ & Sensitivity & Specificity & $\begin{array}{c}\text { AUC } \\
\text { (mean } \pm \text { SE) }\end{array}$ & $\boldsymbol{p}$ value \\
\hline $\mathrm{CRP}(\mathrm{mg} / \mathrm{dl})$ & 11.5 & 66.7 & 80.0 & $0.71 \pm 0.098$ & 0.05 \\
$\begin{array}{l}\text { Lactate } \\
(\mathrm{mmol} / \mathrm{l})\end{array}$ & 14.10 & 70.0 & 80.0 & $0.92 \pm 0.061$ & 0.001 \\
$8-\mathrm{OH}-\mathrm{dG} / \mathrm{dG}$ & 1.3 & 80.0 & 100 & $0.97 \pm 0.032$ & 0.003 \\
\hline $\begin{array}{l}p \text { values are based on AUC as a predictor of survival in sepsis. AUC area under } \\
\text { the receiver operating characteristic curve, CRP C-reactive protein. }\end{array}$
\end{tabular}



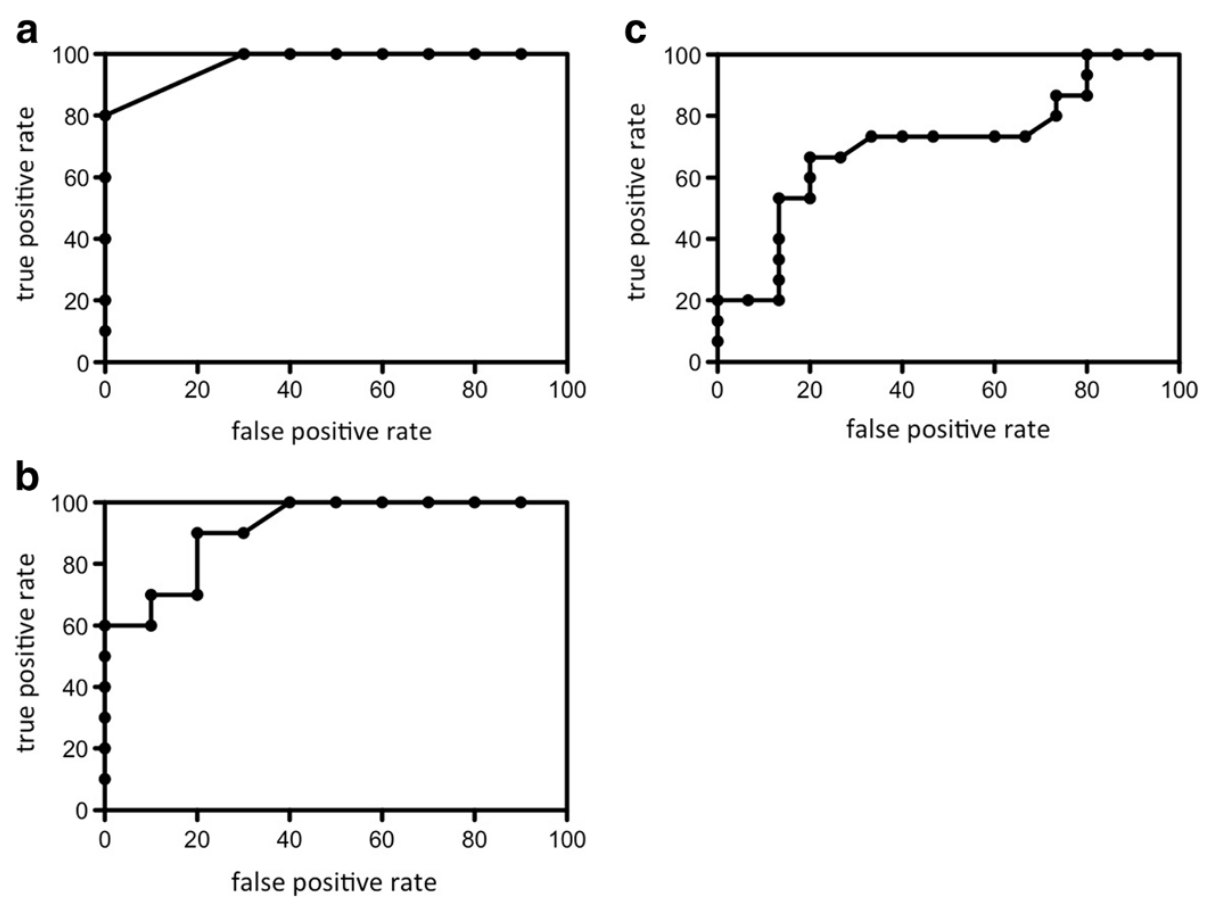

Figure 2 Analysis of the area under the receiver operating characteristic curve. Receiver operating characteristic (ROC) curves of the levels of circulating 8-OH-dG (a), serum lactate (b), and serum C-reactive protein (c) in surviving and non-surviving septic mice.

non-survivors in a mouse model of CLP-induced sepsis. We found that the circulating 8-OH-dG levels, which indicated severity similarly to the lactate levels, were significantly higher in non-survivors than in survivors and were therefore more discriminatory than the CRP level in the ROC curve analysis.

We consider that the levels of lactate and 8-OH-dG were similar in our study because both are produced by mitochondrial dysfunction. However, for indication of severity, 8-OH-dG was slightly superior to lactate for the following potential reasons: First, 8-OH-dG generates oxidative DNA damage easily that may not develop into mitochondrial dysfunction. Furthermore, mitochondrial dysfunction causing hypoxia through the TCA cycle and/or glycolytic pathway abnormality becomes indispensable for lactate generation. Thus, 8-OH-dG may be more easily generated than lactate. Second, the generation of lactate is caused by only mitochondrial dysfunction; however, regardless of mitochondrial dysfunction, 8-OH-dG is generated by not only oxidative mitochondrial DNA damage but also nuclear DNA damage. These findings suggest that $8-\mathrm{OH}-\mathrm{dG}$ was slightly superior to lactate for indicating severity in our study.

However, elevated serum CRP levels are also correlated with increased organ failure [4]. Compared to CRP, the levels of lactate and 8-OH-dG may serve as more sensitive markers to reflect both disease severity and systemic inflammatory responses, possibly because organ injury is not directly reflected in the peripheral blood, as
CRP is metabolized through the liver. Therefore, the CRP level may vary according to the stage of the liver injury in sepsis.

In addition, it should be noted that this study only compared the mortality; however, intracellular ischemic conditions seem to depend on the disease stage. Currently, a limited course of vasopressor therapy indicates reversible tissue hypoxia; however, prolonged vasopressor usage for hemodynamic support is associated with worse lactate clearance [5]. In the clinical course, a significant inverse relationship has been demonstrated between lactate clearance (the resolution of global tissue hypoxia during the first $6 \mathrm{~h}$ ) and non-survivors in severe sepsis and septic shock [6]. Further studies with a long study period are necessary to confirm the relationship of circulating 8-OH-dG with prognosis and therapeutic responses in sepsis-induced multiple organ dysfunction.

\section{Conclusion}

In conclusion, this study showed significantly increased levels of circulating 8-OH-dG in non-survivors in a mouse model of CLP-induced sepsis. The changes of 8$\mathrm{OH}-\mathrm{dG}$ levels in the ROC analysis were found to be similar to those of lactate concentrations, implicating circulating 8-OH-dG in intracellular environmental conditions. We suggest that further research examining the pathogenic mechanisms or novel therapies for severe sepsis should include 8-OH-dG clearance as a measure of prognosis and therapeutic responses. 


\section{Abbreviations}

8-OH-dG: 8-oxodeoxyguanosine; AUC: Area under the receiver operating characteristic curve; CRP: C-reactive protein; DNA: Deoxyribonucleic acid; i.p.: Intraperitoneal administration; ROC: Receiver operating characteristic.

\section{Competing interests}

The authors declare that they have no competing interests.

\section{Authors' contributions}

Dr. JH made substantial contributions to the conception and design, acquisition of data, and analysis and interpretation of data. Dr. JH and Dr. MO participated in the drafting of the article and revised it critically for important intellectual content. Prof. TO and Prof. KK gave final approval of the version to be submitted and any revised versions. All authors read and approved the final manuscript.

\section{Author details}

${ }^{1}$ Department of Emergency and Critical Care Medicine, Kinki University Faculty of Medicine, Nara Hospital, Nara 630-0293, Japan. ${ }^{2}$ Department of Microbiology, Hyogo College of Medicine, Hyogo 663-8501, Japan.

Received: 17 February 2014 Accepted: 11 June 2014

Published: 3 July 2014

\section{References}

1. Nathens AB, Curtis JR, Beale RJ, Cook DJ, Moreno RP, Romand JA, Skerrett SJ, Stapleton RD, Ware LB, Waldmann CS: Management of the critically ill patient with severe acute pancreatitis. Crit Care Med 2004, 32:2524-2536.

2. Abraham $E$, Singer M: Mechanisms of sepsis-induced organ dysfunction. Crit Care Med 2007, 35:2408-2416.

3. Rittirsch D, Huber-Lang MS, Flierl MA, Ward PA: Immunodesign of experimental sepsis by cecal ligation and puncture. Nat Protoc 2009, 4:31-36.

4. Lobo SM, Lobo FR, Bota DP, Lopes-Ferreira F, Soliman HM, Mélot C, Vincent $\mathrm{JL}$ : C-reactive protein levels correlate with mortality and organ failure in critically ill patients. Chest 2003, 123:2043-2049.

5. Levy MM, Macias WL, Vincent JL, Russell JA, Silva E, Trzaskoma B, Williams MD: Early changes in organ function predict eventual survival in severe sepsis. Crit Care Med 2005, 33:2194-2201.

6. Nguyen HB, Rivers EP, Knoblich BP, Jacobsen G, Muzzin A, Ressler JA, Tomlanovich MC: Early lactate clearance is associated with improved outcome in severe sepsis and septic shock. Crit Care Med 2004, 32:1637-1642.

doi:10.1186/2052-0492-2-41

Cite this article as: Hirata et al: Increased levels of circulating

8-oxodeoxyguanosine as a biomarker of sepsis severity in mice. Journal of Intensive Care 2014 2:41.

\section{Submit your next manuscript to BioMed Central and take full advantage of:}

- Convenient online submission

- Thorough peer review

- No space constraints or color figure charges

- Immediate publication on acceptance

- Inclusion in PubMed, CAS, Scopus and Google Scholar

- Research which is freely available for redistribution 